\title{
Design and implementation of virtual environments for planning and building sustainable railway transit systems
}

\author{
M. Chandramouli ${ }^{1}$, B. Huang ${ }^{1}$, T.-Y. Chou ${ }^{2}$, L. K. Chung ${ }^{2}$ \\ \& Q. $\mathrm{Wu}^{1}$ \\ ${ }^{1}$ Department of Geomatics Engineering, University of Calgary, Canada \\ ${ }^{2}$ GIS Research Center, Feng Chia University, Taiwan
}

\begin{abstract}
This article explores the application of virtual reality techniques to rail transit systems from the design and training perspective. We present virtual environments for interactive $3 \mathrm{D}$ visualization of a rail transit station. We describe the design and implementation of some 3D models, which offer a good level of user-interaction and animation within a common rail transit system. The virtual environments for interactive 3D visualization are modeled using Virtual Reality Modeling Language (VRML), a web standard for creating virtual worlds.

Two basic categories of models are discussed in our study. The static 3D models, which are used for planning and design purposes, are based on the object-oriented approach. The dynamic models, which are used for simulation and driver-training purposes, are based on the event-driven approach. Even though a standalone VR application is not capable of a higher level of interaction or animation, using SCRIPT nodes, JavaScript can be embedded in the program to provide additional functionalities. JavaScripting can be used ingeniously to manipulate and control some of the key animation and interpolation nodes to provide powerful functionalities within the VR world. In this study, we integrate the script programming language with virtual reality and elucidate simulations that can be employed for driver training purposes. By employing these visualization and simulation techniques, designers, engineers, planners, and decision-makers can assess the plan beforehand. The virtual worlds serve as immensely useful tools during the design process by helping the designers to position themselves in photo-realistic $3 \mathrm{D}$ immersive environments wherefrom the actual scenario can be viewed and modifications made accordingly.

Keywords: virtual environments visualization, animation, rail transit systems, object-oriented approach, event-driven approach, object linking and embedding.
\end{abstract}




\section{Introduction}

These days, rail stations must cater to the demands of a considerably larger group of commuters with varying needs and demands. Hence, considerable care has to be taken in planning and designing the railway stations. Visualization is a very useful tool that holds immense potential in planning and designing modern rail transit stations. Visualization enables seeing beyond the individual objects or components of the scene and facilitates understanding the entire scene as an interlinked framework. In real world systems, objects do not exist in complete isolation, but are related to each other, either directly or indirectly. Visualization is a tool of immense value that helps to tackle design problems and facilitates finding an optimal solution, which is both time-saving and economical. Visualization enables viewing the constituent elements of a rail -infrastructure framework in whole as well as in parts. This kind of object-based visualization is of significant value to designers, planners and decision makers.

\section{VRML and 3D Visualization}

A wide range of visualization techniques are available as on date. In fact, any pictorial representation can be considered a visualization tool. Today, one term that is synonymous with visualization over the web is 'Virtual Reality'. Much of the 3D visualization for a wide range of applications is done in a virtual space that is often characterized as 'virtual worlds'. The reason they are called virtual worlds is that they are not actually $3 \mathrm{D}$ worlds in real space, but they are digital or cyber worlds that have their own coordinate systems and define a $3 \mathrm{D}$ virtual coordinate space in which applications can be built. The users can navigate within these virtual worlds, interact with the objects, rotate or scale them, and transform them in multiple ways. These virtual worlds facilitate user interaction with the 3D objects and provide a sense of immersion.

Modeling using virtual environments facilitates visualizing the finished product (constructed rail station) before hand. When the designers, planners, and other possible members who are involved in decision-making view such a model, it facilitates non-destructive testing [1]. If, after construction, the decision-makers feel that the location of terminal facilities is not appropriate or the positions of some architectural elements have to be changed for aesthetic purposes, this would involve a lot of wastage of time and resources. However, a 3D immersive virtual environment enables visualizing the station even before it is built and the planners and decision-makers can view and navigate through the model at the convenience of an office desktop and changes can be made without wasting any money. The model can be viewed, discussions held, modifications can be finalized and these can be incorporated before the actual construction can begin. One recent trend is to incorporate public-participation in infrastructure projects. In such cases, the model can be hosted online and members of the public can walk-through the model and suggest possible modifications. Suitable suggestions can be incorporated into the model before actual construction can begin. 


\section{Static 3D models: Rail Transit Systems}

This section focuses on the application of VRML for planning and designing rail transit stations using static models. The basic difference between a static and a dynamic model is that the objects in a static model do not have animation capabilities. Using static 3D models, it is possible to test an application before implementation and virtual worlds can be used to systematically construct rail transit stations [2]. More importantly such environments allow the design of transit stations according to diverse and changeable real-world requirements. The salient advantages of such 3D visual representations are that

- Multiple scenarios can be evaluated

- Infinite viewpoints can be generated

- Decision-makers can view the finished product before hand

- The virtual models are extremely time-saving and economical

- Visibility of signals and various other elements can be tested

\subsection{Planning and design of terminal stations using object oriented approach}

Designing modern railway stations is a complex process and the following important factors must be considered and incorporated into the planning and design process [3]. Today's rail stations are more complicated owing to the following reasons:

- Rail stations handle considerably greater number of trains per day

- Passenger numbers have increased dramatically over the past several years

- Many rail terminals are supposed to cater to multi-modal transport demands

- Stakeholders in station construction includes both public and private parties

- The financial investments in station infrastructure have increased manifold

\subsubsection{Major elements of a rail station}

Some common components of stations, when viewed as a collection of objects, besides platforms and the tracks, are:

- Public Telephones

- Vending stalls and/or Machines

- Toilets

- Ticket halls

- Luggage handling/storage rooms

- Station management room

- Fire-fighting or emergency equipments

- Stairs, elevators, and escalators

This is by no means an exhaustive list, as the station components vary depending on the complexity of operations and the level of commuter service. Nevertheless, in today's scenario stations are in a position to include more and more advanced equipments and technologies that can offer convenient facilities to the commuters. In such a situation the challenge is to properly position the existing infrastructure facilities in an optimal manner to best serve the commuters. 


\subsubsection{Design criteria}

Stations can be defined as the links where the rail transit system establishes contact with the neighbouring regions. Rail terminal stations are also the points where commuters get access to the services and change over to other modes of transport. Therefore, meticulous station design and planning for optimal use of the system is inevitable. The primary objectives in the design and planning of a station must inevitably pay utmost care to include

○ Aesthetic look

- Provisions for emergency management

- Adequate quantity and quality of facilities

The mere provision of space required for the infrastructure cannot satisfy the aforementioned goals. More than the amount of space available, it is the ingenious utilization of this space that determines the efficiency and performance of a station. The consumers i.e. the commuters might have diverse observations spaces [4] in a platform and such views should be able to elicit the amenities available and hence provide a wholesome glimpse of the station. One important consideration while including various amenities in a station should be that the several shops and booths should be carefully located so as not to impede commuter movement, whilst at the same time not located on an island within the station so that commuters who need services urgently can not access them. Currently, station design and approval might involve experts and specialists from diverse backgrounds, such as designers, engineers, technicians, architects, and even economists. In order for the designers and architects to be able to explain their schema to all the members clearly, they need to make use of a tool such as visualization. 3D visual worlds are excellent tools for communication and can be easily understood by a broad spectrum of people.

\subsection{Station design using VRML}

VRML adopts a hierarchical structure for describing 3D worlds. Visualizing the world as a group of objects within a structural framework facilitates modeling real-world scenarios better [2]. Typically, the elements mentioned earlier under section 3.1.1 can be found in a rail station. The station can be considered the root object that is at the top of the hierarchy, under which all other objects fall. There can be further ramifications depending on the object's complexity. For instance, platform is a child of 'Rail Station' and 'Ticket Hall' is a child of 'Platform' object (Figure 1).

There are several advantages of such grouping of elements composing a scene in the form of objects that have group or parent-child relationships. First and foremost, the overall organization becomes lucid and basically no element, however large or small, is left out. This facilitates quantity estimation greatly and also aids locating system components within a huge framework.

Another prominent feature offered by the object-oriented approach is that of 'reusable software objects' (Figure 2). Quite frequently, a single element is used more than once within a parent or group object. For instance, wheels of locomotives, sleepers used in the track system, inventory elements such as 
lamps, signposts, furniture elements etc. In such a case, the object that occurs repetitively can be defined just once and instances of the object can be used subsequently.

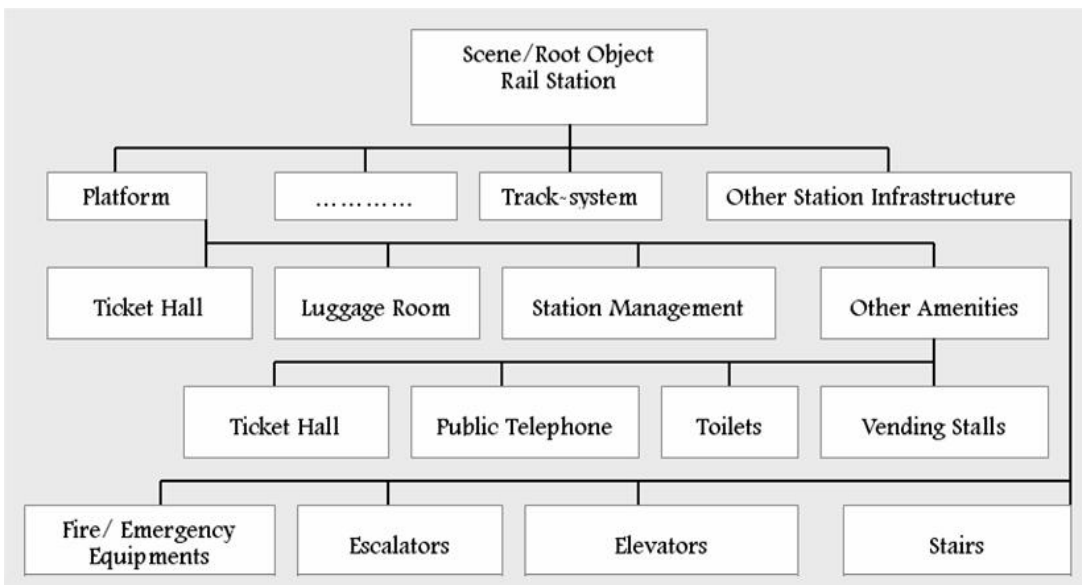

Figure 1: Hierarchical grouping of Station object.

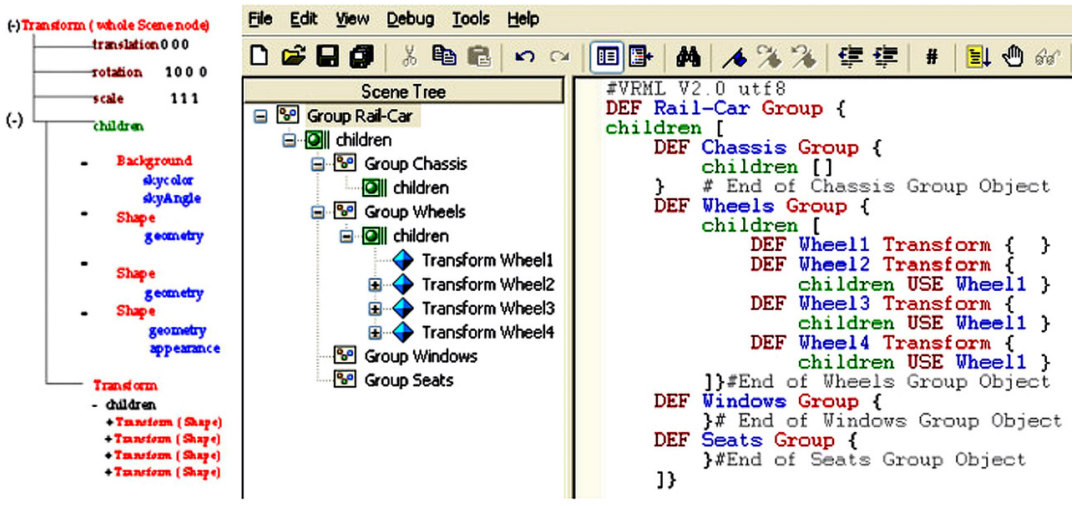

Figure 2: a. Scene Tree structure showing 'Parent-Child' relationships. b. Hierarchical grouping of station elements.

Every component within a rail terminal has its unique function. For instance, the ultimate objective of signboard is to convey relevant and useful information to the commuters. This is lost if a signboard is improperly positioned or is in a condition wherein reading any information from it is difficult. Particularly, in today's scenario wherein international tourists constitute a considerable chunk of commuters in metropolitan rail station, signboards are a key source of information. Virtual worlds serve as an excellent means to view the model of the station and its components from numerous perspectives, and optimally positioning the signboards. Similarly, during the planning stage, the various other elements can be placed in different positions at different locations and thus 
can be seen from different perspectives and even from different heights. This will facilitate locating intruding objects or judging visibility.

\subsection{User interface for multi-perspective and multi-scenario visualization}

Figure 3 illustrates a VB powered GUI (Graphical User Interface) that facilitates multiple-scenario visualization with interactive functionalities. By means of OLE (Object linking and Embedding), the various virtual worlds are embedded and linked on a common interface. Each scenario depicts a different configuration of the same rail station, which can be a simple re-organization of the elements in the previous scenario or a total rearrangement of the station components.

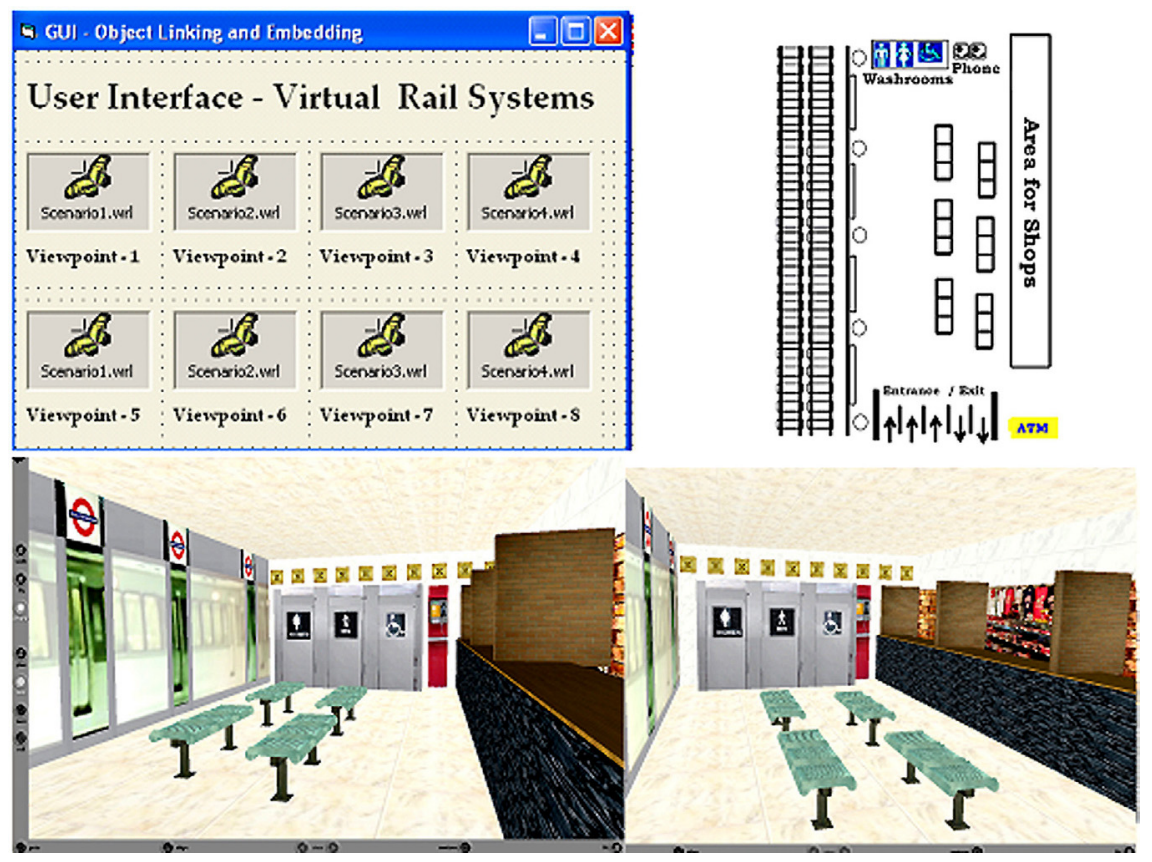

Figure 3: a. Visual basic GUI with Object linking and Embedding (OLE) functionality. b. Rough Layout. c. Viewpoint 1. d. Viewpoint 2.

Such virtual environments can also be used to create sophisticated rail car and locomotive models [5]. A very high level of detail can be built into the design of such 3D models and the dynamic functioning of the locomotive components can be visualized. Under real-world circumstances, it is practically impossible to get to the level of the individual components each and every time when it is required to visualize changes made. Using VRML highly detailed models capable of dynamic behaviour can be generated and more importantly these models are scalable. More importantly, the training can be incorporated even when the model is being designed. Some other key functionalities that can be added into the virtual worlds are described in Figure 4. The rail station model shown above is composed of 'Inline' nodes, described subsequently, as well. 


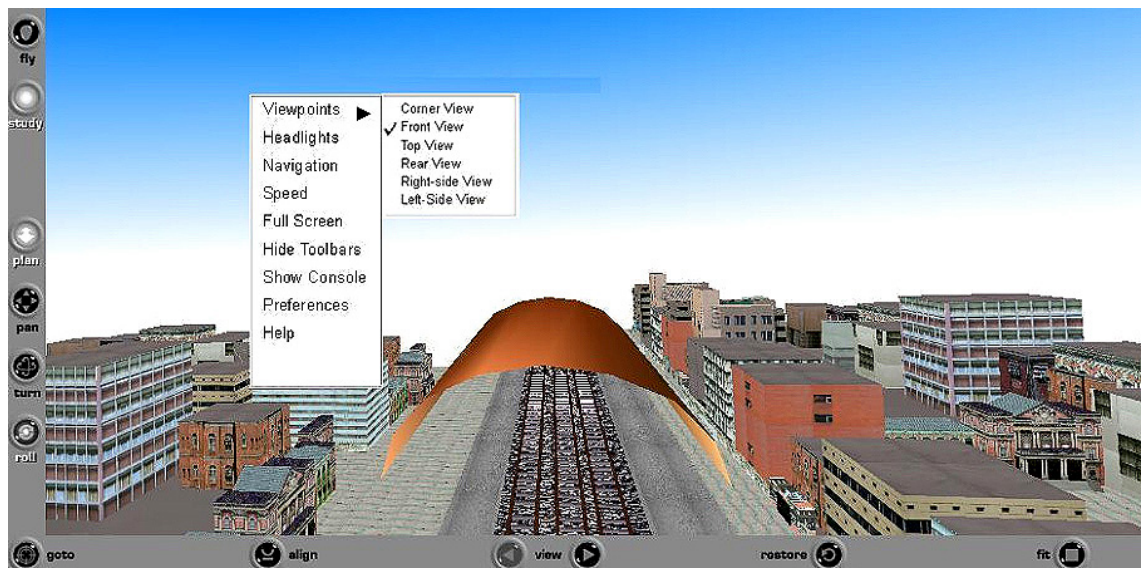

Figure 4: $\quad$ VRML world with multiple viewpoints.
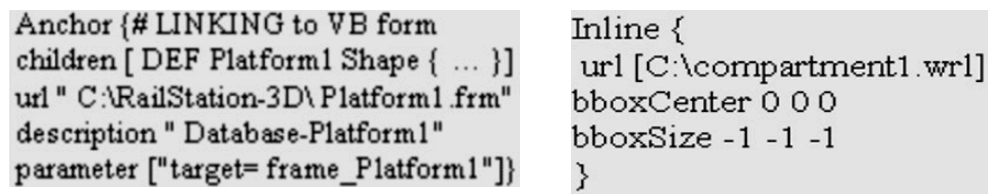

Figure 5: Anchor node and Inline node functionalities.

Once the 3D component of the model generation is done, any descriptive information or annotation can be associated with the corresponding object. This may include any type of information as the application demands. For instance, an application might demand the linking of GPS positional information and another application might require facilitating user-input by means of visual basic forms. This is done by means of the 'Anchor Node' construct shown above. Similar to the Anchor node functionality, another node that is used in the construction of databases or 3D worlds is an Inline node illustrated above. A VRML scene can be divided into a set of files. This not only simplifies the design of the VR world, but also facilitates reusing parts of the world already built in one scene in many other worlds. In the case of the railway station, a set of shapes that draw a train or compartment are grouped together and stored under a single node. This group node can be given a specific name, say 'compartment1', and reused later in another world. The Inline node permits the specification of a URL from where a specific file or data can be retrieved and reused in another file [7].

\section{Dynamic models: simulation systems for training}

This section focuses on the development of dynamic simulation models that can be used in the process of driver-training. Simulation is also a very powerful tool for collaboration, particularly with the practically unlimited connectivity and access provided by WWW. Manufacturing, assembling, and testing a prototype locomotive engine or rail car is a laborious and time-consuming task indeed. It is also an expensive process. We see that there are several aspects to be considered 
at several stages ranging from product design and assembly to final implementation. Some vital factors include:

- $\quad$ Training to be imparted to those involved in manufacturing and assembling

- Replicating product movement i.e. functioning of locomotive engine

- Imparting training to drivers who will be operating the trains

When using such simulations to impart training, the participants can get a 'feel' of the on-site circumstances and experience the practical difficulties without being exposed to the associated risks. A mistake on the field, for instance, a wrong maneuver while driving a train or a careless step during assembly might even be fatal at times. However, using simulation for training eliminates the hazards inherent in such processes and thus facilitates a safe training environment. Moreover, training involves both aspects that are straightforward and can be easily understood and those that are obscure and are rather difficult to grasp. The understanding of such tricky and subtle aspects is imperative to gain thorough understanding of real-world systems that inherently involve risks to public property and lives. Simulation is an effective tool under such circumstances as, once generated, the simulation models can be run as many times as required to familiarize the trainees thoroughly with the system [6]. Trainers do not need to be repetitively hired to impart training and no additional money is required to regenerate the training scenario.

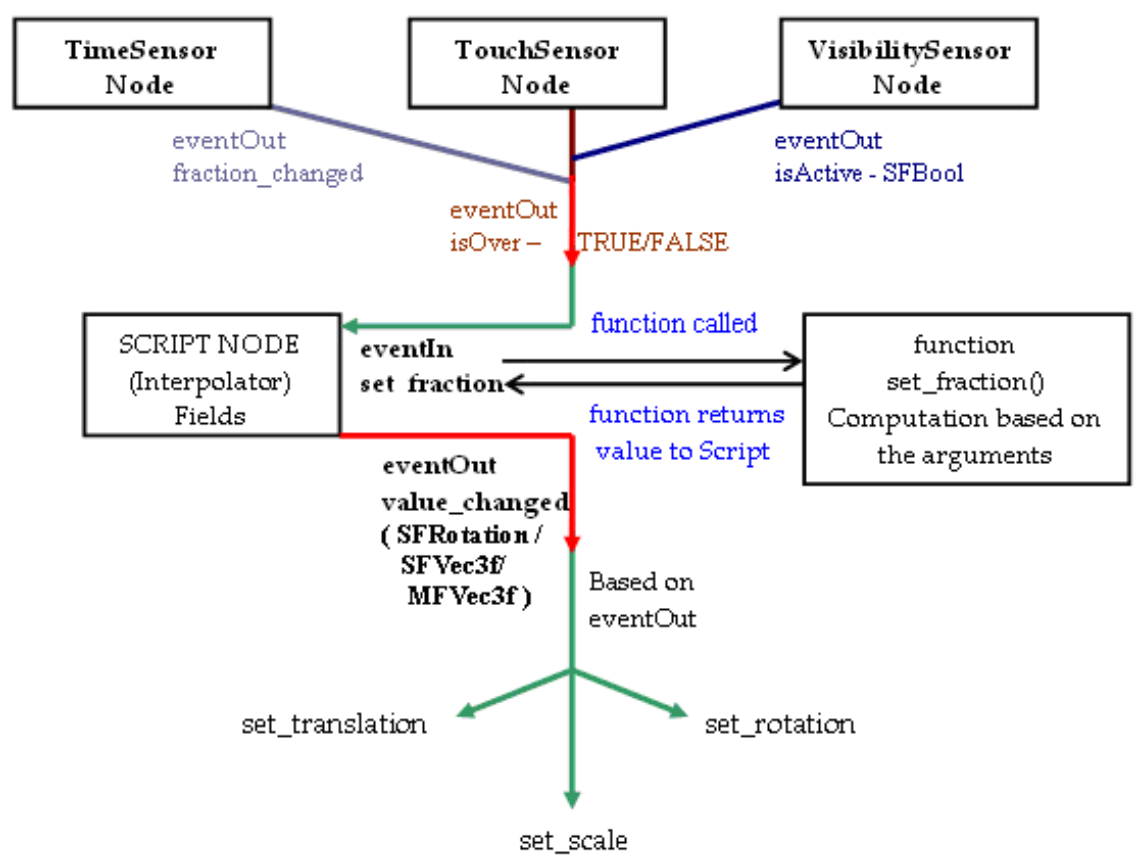

Figure 6: The flow of events among the nodes in an animation circuit. 


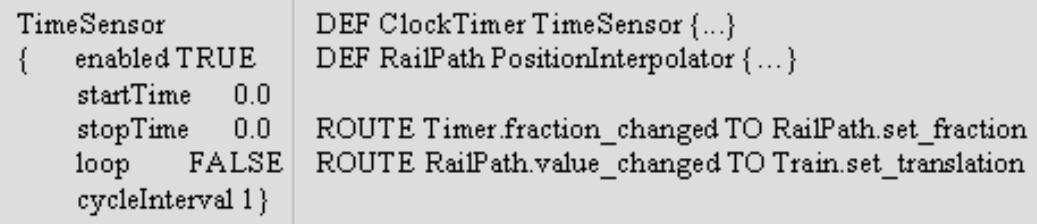

Figure 7: TimeSensor node and the ROUTE function for train movement.

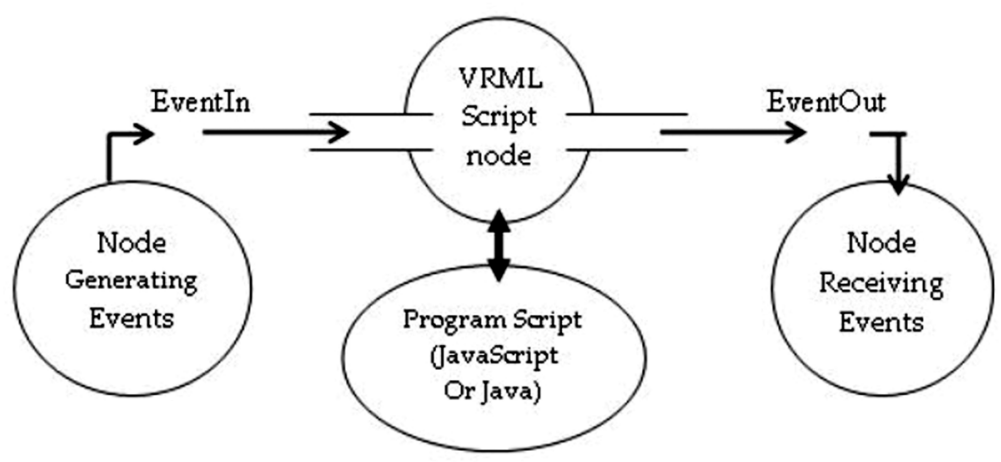

Figure 8: $\quad$ Flow of events among the nodes and the SCRIPT program.

\subsection{Designing dynamic models using event-driven approach}

Having seen the advantages of using 3D simulations, the discussion now focuses on the actual animation framework within virtual worlds. As mentioned in the previous section, using JavaScript or Java in conjunction with VRML 2.0 provides animation and interaction capabilities to the VR world.

The Script node in VRML calls upon a JavaScript linked with the node to function within the browser. While this refers to the internal control of the scene from within a node in the VR code, the EAI (External Authoring Interface) allows manipulating the scene externally. Movements within a VR world are brought about by animating position, orientation, or scale of the coordinate systems in which the train objects exist. A clock is created by means of the TimeSensor node and the changes can be described using the PositionInterpolator and OrientationInterpolator nodes. In this case, the simulation depicts the movement of a train along rail paths between stations or along test routes. The path is a linear or a curvilinear path. The position along this linear or curvilinear path is specified using PositionInterpolator and OrientationInterpolator nodes.

The events must be transmitted from the TimeSensor to the PositionInterpolator and from the PositionInterpolator to the Transform node. The ROUTE statement is used to link event from one node to another node. The output event from PositionInterpolator, value changed is routed to the translation field of the Transform node containing the 'Train' object. As 
animation progresses, the clock continues to send changed time values (SFTime) and correspondingly new positions are chosen and output. This serves as the input for the Transform node of the 'Train' object, thus causing the node's coordinate system to undergo corresponding transformation (translation, scaling, and/or rotation). As a result, the 'Train' movement or simulation is achieved (Figure 9).

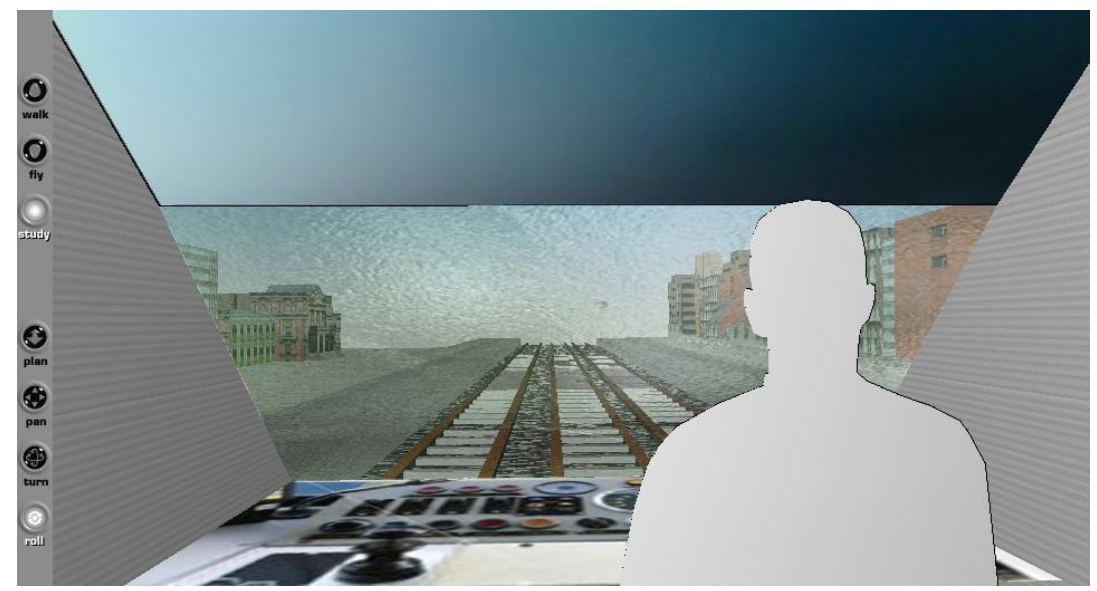

Figure 9: $\quad$ Simulation training for drivers using virtual worlds.

\section{Discussion and conclusion}

The primary aim of this study was to explore the use of virtual reality tools and techniques in building sustainable railway transit systems. This study discussed the process of a prototype model generation using VRML and animating the scene using a SCRIPT node and a scripting language, JavaScript. Even though the conventional drawings and other $2 \mathrm{D}$ representations may provide an idea of the place being designed, these cannot substitute a 3D view. Present rail station design and locomotive modeling efforts are far more complex when compared to those a couple of decades ago. Hence, it is imminent 3D data be viewed in their 3D forms in order to gain a better insight. Also, during the design and planning process, it is common to come across a situation wherein there is a need to visualize a future scenario.

This study explained with examples and prototype VR models the design and construction of railway transit systems and also the use of such systems to provide training to drivers. This study is just a beginning in the efforts to utilize the immense potential of virtual worlds in railway transit designing and planning. Future research may focus on the design of innovative locomotive and rail car designs and the creation of advanced simulations that impart state-of-theart training at extremely low cost without wasting any resources. 


\section{References}

[1] Doyle, S., Dodge, M., \& Smith, A., (1998): The potential of web-based mapping and virtual reality technologies for modeling urban environments. Computers, Environment and Urban Systems, 22: 137 155.

[2] Worboys, M. F. (1994): Object-oriented approaches to geo-referenced information, International Journal of Geographical Information Systems, 8(4), pp. 385-399.

[3] Section 4 Design guidelines Railway Design guidelines and criteria November 2005.

[4] Michael Meillard, 1995. Reinventing the railway station, Japan Railway and Transport Review 1995.

[5] Jepson W, Ligget R And Friedman S (1995), An Environment for Real time urban simulation, I 1995 Symposium On Interactive 3d Graphics, ACM.

[6] Sheppard, S.R.J. 1989. Visual Simulation: A User's Guide for Architects, Engineers, and Planners. Van Nostrand Reinhold, New York.

[7] Ames, L.A, Nadeau, D.R., Moreland, J.L., 1996, VRML 2.0 Sourcebook, John Wiley \& Sons. 